\title{
El ecosistema de aprendizaje del estudiante universitario en la post-pandemia. Metodologías y herramientas
}

\author{
Francisco Charte, Antonio J. Rivera, Javier Medina y Macarena Espinilla \\ Departamento de Informática, Universidad de Jaén. \\ \{f charte, arivera, jmquero, mestevez\}@ujaen.es
}

\begin{abstract}
Resumen La transición de una modalidad de enseñanza tradicional y presencial a una de tipo remoto, provocada por el confinamiento a raíz del coronavirus SARS-CoV-2, ha implicado cambios que han debido realizarse de manera acelerada y que afectan no solo al desarrollo de las clases, sino también a las actividades prácticas en laboratorio, de comunicación y de evaluación de las competencias de los estudiantes. En este trabajo exponemos cómo hemos afrontado dicha transición en asignaturas del área Arquitectura y tecnología de computadores en la Universidad de Jaén, así como la planificación que hemos llevado a cabo para el próximo curso 2020/2021 ante el nivel de incertidumbre sobre cómo se desarrollará.
\end{abstract}

Palabras clave: metodologías de aprendizaje, aprendizaje activo, aprendizaje basado en proyectos, modelos de evaluación.

\begin{abstract}
The transition from a traditional and face-to-face teaching modality to a remote type, caused by the confinement due to the SARS-CoV-2 coronavirus, has implied changes that have had to be made in an accelerated way and that affect not only the development of the classes, but also the practical activities in the laboratory, communication and evaluation of the students' competences. In this paper we explain how we have faced this transition in subjects of the area Computer architecture and technology at the University of Jaén, as well as the planning we have carried out for the next academic year 2020/2021 in view of the level of uncertainty about how it will develop.
\end{abstract}

Keywords: learning methodologies, active learning, project-based learning, assessment approaches

You cannot get educated by this self-propagating system in which people study to pass exams, and teach others to pass exams, but nobody knows anything. You learn something by doing it yourself, by asking questions, by thinking, and by experimenting. Richard Feynman.

\section{Introducción}

La situación generada por la expansión del coronavirus SARS-CoV-2 a nivel mundial, especialmente en aquellos países en los que ha explotado con mayor virulencia 
como es el caso de España y, en consecuencia, ha implicado medidas más estrictas de confinamiento, ha tenido un gran impacto en el desarrollo de la actividad universitaria. El personal docente es consciente de los obstáculos que ha encontrado para llevar a cabo la actividad académica mediante tecnologías que, en general y hasta ahora, no eran las que empleaba habitualmente para impartir la docencia. A esto se han sumado otros aspectos también destacables, como el entorno en que ha tenido que desarrollarse la actividad: el doméstico, no siempre adecuado para ella, o los conocidos problemas de conciliación, que quedan fueran del ámbito de estudio de este trabajo.

$\mathrm{Al}$ otro lado de la actividad docente se encuentra el estudiante, cuyas circunstancias se han visto igualmente afectadas por la citada situación. El ecosistema de trabajo habitual, que se desarrollaba entre aulas: laboratorios, salas de estudio y la comunicación directa con profesores y compañeros, se ha trasladado a un entorno con muchos menos recursos físicos (equipamiento, bibliografía, espacios de trabajo y estudio) y que plantea nuevos desafíos para mantener un contacto fluido y cercano con el resto de protagonistas determinantes en el proceso de aprendizaje personal.

El cambio acelerado y no planificado, de un escenario normal a uno de confinamiento, se produjo a mitad del segundo cuatrimestre del curso académico 2019/2020 y exigió una búsqueda de rápidas soluciones y adaptación que, permitiendo continuar con la actividad lectiva, presentaron no pocos obstáculos. La alternativa más empleada, hasta donde sabemos por nuestro entorno, ha sido la transición de una enseñanza presencial a una de tipo remoto (que no virtual) manteniendo el esquema general de funcionamiento: clases magistrales de forma síncrona, realización y entrega de prácticas mediante los mecanismos habituales y evaluación tradicional a través de examen, cambiando la modalidad escrita por la ofrecida por el LMS (Learning Management System) correspondiente.

Finalizado el curso, nos enfrentamos a la necesidad de llevar a cabo una planificación para el curso 2020/2021, con un alto nivel de incertidumbre. En general, las universidades han planteado diferentes escenarios potenciales: i) el de la normalidad absoluta, que todos deseamos pero a priori parece poco probable; ii) el de la denominada nueva normalidad, que conlleva una reducción en la tasa de ocupación de aulas y laboratorios conjuntamente con medidas de distanciamiento y de tipo sanitario (desinfección de instalaciones, uso de mascarillas, etc.), y finalmente, iii) el escenario de un nuevo confinamiento general si la situación se agrava. En marzo de 2020 no existía ningún tipo de previsión, a nivel estatal, autonómico o de centros educativos, sobre cómo debería afrontarse una situación inesperada como la que se generó en aquel momento. Ahora, no obstante, la experiencia adquirida debe servirnos para enfrentar el próximo curso de una forma más previsible.

Con este artículo pretendemos transmitir a la comunidad educativa nuestra experiencia en la adaptación de asignaturas con docencia por parte del área de Arquitectura y tecnología de computadores de la Universidad de Jaén, como son Programación y administración de redes y Sistemas empotrados y ubicuos, tanto en la primera fase de urgencia como en previsión de los escenarios del próximo curso, haciendo especial énfasis en las herramientas y metodologías que determinan el ecosistema de aprendizaje en el que ha de vivir el estudiante. 


\section{Obstáculos en el proceso de aprendizaje clásico}

La metodología de enseñanza tradicional o clásica, todavía la más empleada en términos generales [1], tiene su pilar fundamental en la clase magistral. El centro es el profesor que, mediante la vía oral y apoyándose en algunos recursos, esencialmente la pizarra y/o diapositivas, transmite su propio conocimiento a unos oyentes, los estudiantes, que salvo excepciones adoptan el papel pasivo del proceso. En un contexto en el que la fuente del conocimiento, la materia que se estudia, ya no es exclusiva del docente como ocurriera en el pasado sino que está a disposición inmediata a través de Internet, el enfoque previo no tiene necesariamente que ser la mejor alternativa.

Por ello, la comunicación entre estudiante y profesor juega un papel fundamental, siendo más o menos fluida según los casos, así como la interacción entre los propios estudiantes, tanto durante como después de la clase. La primera permite al estudiante resolver aspectos que le planteen dudas, en primer término, y al profesor presentar a los estudiantes cuestiones a analizar y problemas a resolver en relación al tema de estudio. La comunicación entre estudiantes, que se produce tanto en la clase como fuera de esta a lo largo de todo el curso, puede llegar a ser incluso más importante. El apoyo entre pares es en muchas ocasiones el método preferente para solventar dudas, más empleado que las tutorías con el profesorado.

El cambio brusco a una enseñanza remota ha levantado obstáculos importantes para el normal desenvolvimiento de este tipo de clases, centradas en la explicación facilitada por el profesor. En principio identificamos los siguientes obstáculos:

1. Dificultades para seguir las clases en el horario establecido al no contar el estudiante con los recursos adecuados: ordenador, servicio de acceso a Internet, etc. En muchas ocasiones esos recursos son compartidos por varios miembros de la entidad familiar, de forma que si un progenitor los tiene que usar para desarrollar su actividad laboral no pueden estar a disposición del estudiante.

2. Fácil desconexión de la atención del estudiante durante la clase, ya que mientras escucha, al estar delante de un ordenador, puede estar realizando otras tareas que actúan como distracción.

3. Falta de comunicación fluida con el profesor impuesta por los medios empleados. En general los estudiantes evitan usar el micrófono para plantear sus preguntas verbalmente, empleando en su lugar una ventana de mensajes (chat) que fuerza al profesor a estar vigilando este recurso y reaccionando a él al tiempo que se desarrolla la clase.

4. Interrupción de la comunicación habitual con los compañeros que, como se apuntaba antes, constituye posiblemente el principal pilar de apoyo para muchos estudiantes. La interacción personal puede, hasta cierto punto, ser complementada por la comunicación digital, siempre con las limitaciones que impone el primero de los obstáculo mencionados.

Uno de los problemas fundamentales en el cambio a remoto es la equidad en cuanto a recursos y entorno de trabajo de los estudiantes, aspectos fuertemente dependientes del contexto familiar [2] como se indica en el primer obstáculo. En general, los estudiantes de titulaciones técnicas y, en particular, relacionadas con la informática, disponen de un ordenador y de conexión a Internet, así como de teléfono móvil, pero el entorno familiar 
puede imponer limitaciones de espacios, horarios de uso del ordenador (compartido), etc. La denominada brecha digital, que se incrementa y se une a la brecha social en esta situación [3], ha de ser contemplada para no dejar a ningún estudiante atrás.

Frente a esta metodología de enseñanza tradicional, y los obstáculos que plantea la situación actual, es preciso definir una metodología del aprendizaje, diseñando un entorno virtual centrado en el estudiante [4], con ideas creativas que le resulten valiosas para desarrollar su actividad. Hay que partir de que aprendizaje en sí mismo, como proceso de adquisición de conocimientos por parte de las personas, no es un lugar, no es un aula, sino que es un camino que ha de recorrerse y para el que se necesita la guía adecuada. El docente es el que ha de liderar dicho proceso, proveyendo al estudiante de los recursos, herramientas de trabajo, mecanismos de seguimiento y mejora, etc. El uso de ideas creativas que mejoren el ecosistema de aprendizaje del estudiante, en esto consiste la innovación docente, redundará asimismo en una mejora de los resultados académicos, no tan dependientes de la presencialidad en clase.

\section{Adaptación de las metodologías}

La necesaria adaptación de la metodología clásica de la enseñanza a una metodología del aprendizaje, fundamentalmente aprovechando el universo de posibilidades que ofrece el mundo digital, es algo que ya estaba experimentándose desde hace tiempo [5]. La situación de no presencialidad lo que ha hecho, en cierta manera, es acelerar ese cambio y, sobre todo, nos ha permitido conocer cuáles son las necesidades y los problemas existentes para poder completar dicha adaptación de forma satisfactoria.

Para comenzar, hemos de tomar en consideración que los medios digitales no sustituyen por completo a las actividades presenciales, sino que las complementan facilitando aspectos como el acceso a los recursos (biblioteca digital, herramientas virtuales, etc.), la comunicación no síncrona (a través de foros, correo electrónico o redes sociales), el trabajo colaborativo entre los estudiantes, la realización, entrega y evaluación de trabajos, etc. En caso necesario el mundo de lo digital, con las herramientas apropiadas, puede llegar a convertirse en el núcleo del proceso de aprendizaje si las circunstancias lo requiriesen.

También es preciso observar que el simple cambio de la presencialidad en clase por una presencialidad remota, a través de un sistema de vídeoconferencia, de manera síncrona y empleando los mismos métodos que se usarían en clase, no aporta innovación alguna respecto a la metodología tradicional. La verdadera transición consiste en que el docente pase de ser solo un instructor a instituirse en el interlocutor cualificado [6] para llevar a cabo el proceso de aprendizaje de manera satisfactoria, proveyendo al estudiante los recursos y métodos apropiados para que este construya un ecosistema de aprendizaje lo más óptimo posible y adecuado a sus necesidades y, lógicamente, desempeñando el papel de autoridad competente, con los conocimientos necesarios, en aspectos como la evaluación de las competencias adquiridas por el estudiante.

\subsection{Por qué es preciso cambiar las metodologías}

El tiempo compartido entre profesor y estudiantes a lo largo de un cuatrimestre por asignatura, no contabilizando el dedicado a realización de prácticas en laboratorios, es 
muy limitado. En general se reduce a 30 horas repartidas a lo largo de 15 semanas, típicamente con dos clases de una hora a la semana, a las que se sumaría el tiempo de atención individual en tutorías que suele ser mucho menor. ¿Tiene sentido dedicar ese valioso tiempo, al completo, a mostrar diapositivas de una presentación explicando conceptos que el estudiante podría leer por sí mismo?

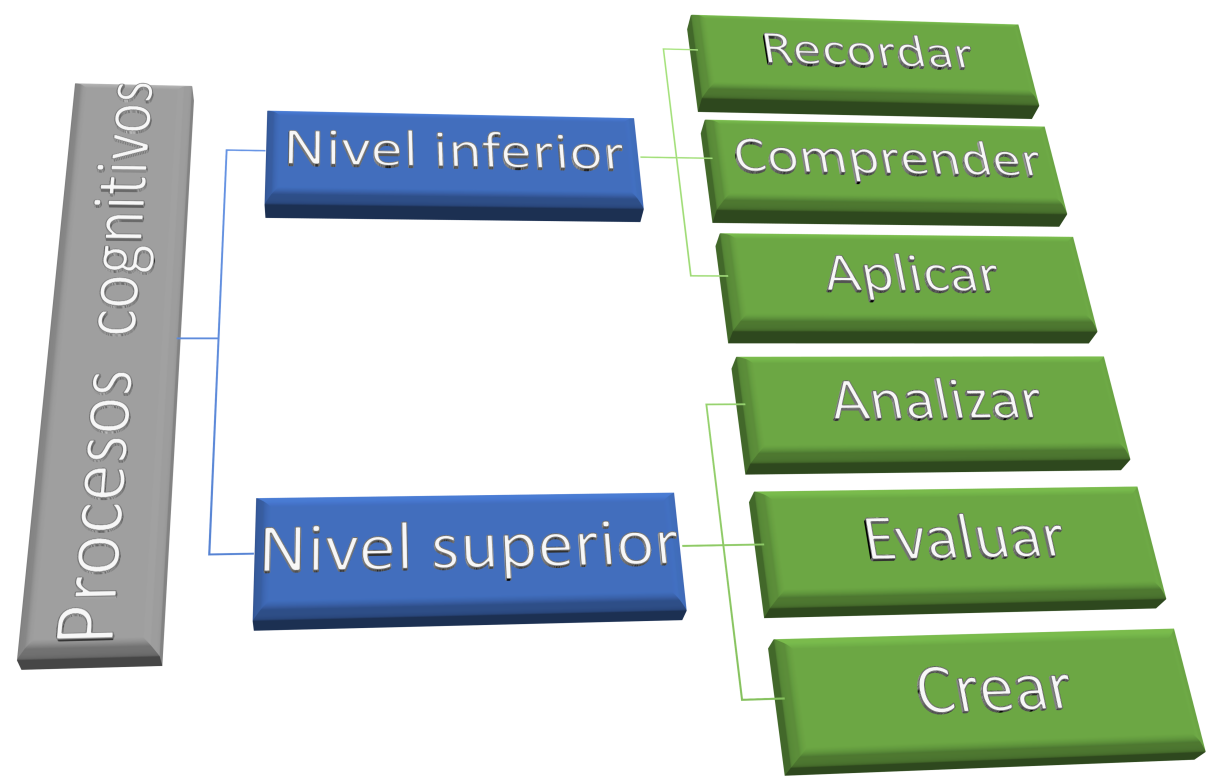

Figura 1. Interpretación actualizada por Anderson de la taxonomía de Bloom.

Atender pasivamente a una explicación, limitándose a ver y escuchar con el único objetivo de adquirir conceptos y recordarlos, es una actividad de bajo nivel cognitivo atendiendo a la taxonomía de Bloom actualizada por Anderson [7] (véase la Figura 1). El proceso de más bajo nivel consiste únicamente en recordar datos y hechos, sin siquiera llegar a comprenderlos, tarea que recae en el siguiente nivel. Ambos niveles implican acciones que el estudiante puede llevar a cabo por sí mismo sin necesidad de mucha ayuda, como son el repaso repetitivo de datos a recordar o la comprensión básica de textos con definiciones. En cuanto al tercer proceso de los considerados de bajo nivel, consistente en aplicar los conocimientos adquiridos en los dos previos para resolver problemas, es el que suele llevarse a cabo durante las sesiones prácticas en laboratorio.

Dejar las tareas con menor nivel cognitivo para el trabajo autónomo del estudiante, en lugar de emplear en ellas el poco y valioso tiempo que se comparte con el profesor, permite dedicar ese tiempo a las tareas de más alto nivel: analizar, evaluar y crear. 
Estos procesos cognitivos implican el uso de metodologías activas del aprendizaje [8], tales como el aprendizaje basado en proyectos [9] y la resolución de problemas [10], la evaluación y el debate de temas, invirtiendo el enfoque de manera que los estudiantes analizan el tema y posteriormente se evalúa y discute en clase, en lugar de ser explicado en el tiempo de clase y posteriormente discutido entre los estudiantes. La creatividad, a través de la investigación de la materia de estudio, recopilación y síntesis de ideas, etc., es también una parte valiosa en el aprendizaje, exigiendo el más alto nivel cognitivo.

\subsection{Qué metodologías podemos emplear}

En la adaptación del proceso de enseñanza/aprendizaje deberíamos siempre conducirnos por la evidencia, por aquello que está constatado que funciona en los estudios publicados en la literatura especializada. El mayor problema que hemos de afrontar es la inmensa cantidad de estudios y propuestas existentes, resumidos a su vez en cientos de meta-estudios. Afortunadamente, Hattie [11] resume en su libro Visible Learning ${ }^{1}$ quince años de trabajos, un total de 913 meta-análisis que recogen más de 60000 estudios (en la edición de 2012), y nos ofrece una valiosa guía sobre qué actividades tienen un efecto positivo/negativo en el proceso de aprendizaje, considerando en torno a 200 aspectos diferentes y su impacto de acuerdo a un barómetro de influencia. Un estudio de la aplicación de esas técnicas a la educación superior es abordado por el propio Hattie en [12].

De entre esos aspectos, una buena parte de los que tienen mayor influencia positiva en el proceso de aprendizaje por parte del estudiante están relacionados con metodologías bien conocidas, como las de aprendizaje activo [8] o el aprendizaje basado en proyectos [9]. En el top $10 \%$ de actividades encontramos temas como las herramientas de autoevaluación [13], los debates de temas en clase, tomar en consideración la realimentación por parte del estudiante en el estudio de la materia, que el docente defina claramente el rendimiento esperado por parte de los estudiantes, el uso del aprendizaje invertido [14] o el uso de programas creativos [15].

Llama la atención que el aspecto que más parece influir en general sea precisamente el conocimiento por parte de los estudiantes de la estimación que el profesorado hace sobre las metas y competencias que deben alcanzarse, así como la evaluación [16] continuada, tanto autónoma como supervisada, a lo largo del curso. La intensificación de la evaluación, de forma que los estudiantes saben en todo momento dónde se encuentran en su proceso de aprendizaje y los profesores conocen la situación de cada estudiante, conlleva aspectos positivos para ambas partes. Los estudiantes se sienten estimulados al conocer su evolución gradual en la materia, mientras que al docente le permite detectar estudiantes con problemas y afrontar así el reto de una enseñanza más individualizada aunque esto no siempre es posible.

Es preciso hacer notar que ninguno de los aspectos citados hasta ahora son exclusivos para una enseñanza a través de medios digitales, sino que son perfectamente aplicables a la clase presencial. Precisamente el uso de este tipo de metodologías, que incluyen trabajos de autoevaluación, desarrollo de proyectos, discusión de temas y un

$\overline{1}$ https://visible-learning.org/ 
enfoque creativo [15] en la realización de trabajos, son las menos afectadas por el cambio de presencial a remoto. Son metodologías que, en general, tienden a incrementar la autonomía del estudiante que, como se apuntaba anteriormente, realizar las tareas de más bajo nivel cognitivo sin necesidad de supervisión, mientras que en las horas de clase compartidas entre profesor y estudiante, ya sea en clase o por vídeoconferencia, se abordan de manera supervisada las de más alto nivel.

\subsection{El aprendizaje activo a lo largo del curso}

Se ha demostrado [8,17] que la adquisición de conocimientos por parte del estudiante no mejora mediante el estudio reiterado de los contenidos, independientemente de que estos se faciliten en formato escrito o audiovisual. Asimismo, la concentración del estudio en un periodo corto de tiempo, en lugar de realizarse espaciadamente a lo largo del cuatrimestre, también resulta menos eficiente. Sin embargo, esta es la técnica empleada usualmente por buena parte de los estudiantes: la lectura repetitiva de diapositivas y/o apuntes de la asignatura cuyo examen abordarán en breve.

En la actualidad, muchos estudiantes presentan una actitud relativamente pasiva respecto al aprendizaje durante el periodo lectivo, entendiendo como tal el previo a la realización de pruebas de evaluación. A partir de la observación durante las clases teóricas y prácticas, así como a través del análisis de accesos a los recursos en línea (plataforma de docencia, vídeos, etc.) y de la frecuencia de uso de las tutorías, se constata que el estudio activo de la materia no se inicia hasta pocos días antes de la realización de pruebas de evaluación: exámenes, presentación de trabajos, etc. Antes, durante el cuatrimestre, los estudiantes son, en general, oyentes pasivos, siendo reducida la participación en clase, toma de apuntes, asistencia a tutorías, etc. Sin embargo, el cambio de este rol pasivo en la universidad puede ser motivado hacia uno activo introduciendo nuevas metodologías docentes [18,19].

Como conductores del proceso de aprendizaje, el objetivo de los profesores es buscar vías para incrementar la implicación de los estudiantes en dicho proceso, mejorando tanto los resultados de evaluación como el conjunto de conocimientos adquiridos por los mismos. Es necesario modificar la metodología de estudio citada, extendiendo el periodo en que se lleva a cabo e introduciendo técnicas que induzcan la actividad por parte del estudiante. Es sabido que las técnicas de autoevaluación funcionan generalmente bien junto al aprendizaje autónomo del alumnado [20], por lo que a priori poner recursos de este tipo a disposición de los estudiantes empleando alguna plataforma sería positivo.

Esta realidad fue la que llevó a los autores a iniciar un proyecto de innovación docente, descrito más adelante, con un doble objetivo: facilitar una plataforma centrada en el estudio autónomo y autoevaluación para estimular el aprendizaje activo por parte de los estudiantes, por una parte, y que asimismo sirviese a los profesores para conocer con detalle el uso de los recurso ofrecidos conjuntamente con el rendimiento obtenido a lo largo del tiempo, por otra.

\section{Adaptación de urgencia y planificada: nuestra experiencia}

El segundo cuatrimestre del curso 2019/2020 se encontraba aproximadamente a mitad de camino cuando el sábado 14 de marzo se activó el estado de alarma (según 
R.D. 463/2020, publicado en BOE $\mathrm{n}^{\mathrm{o}} 67$ de 14/3/2020) y, en consecuencia, quedaron en suspenso las clases presenciales en todos los niveles educativos, incluyendo el universitario.

En aquél momento los autores tenían en marcha el proyecto de innovación docente descrito en la Sección 4.1, mediante el cual parte de los estudiantes tenían acceso a un conjunto de recursos, vídeos y herramientas de autoevaluación, que abarcaban un conjunto limitado del temario. De manera inmediata, durante ese primer fin de semana y los días posteriores, se puso en conocimiento de los estudiantes las herramientas que se emplearían para continuar el seguimiento de las clases según la programación establecida, inicialmente con una herramienta de vídeoconferencia y la metodología habitual. Sobre esta, no obstante, fue preciso ir introduciendo adaptaciones con cierta premura, entre las que destacan las siguientes:

- Virtualización de las herramientas empleadas en el laboratorio con el objetivo de permitir la continuación de las actividades prácticas.

- Creación y publicación de vídeo-tutoriales [21] que guiasen a los estudiantes en la transición del trabajo en laboratorio al uso de las herramientas virtualizadas.

- Ampliación de tutorías grupales [22], demandadas por los propios estudiantes, para suplir la falta de comunicación en laboratorio que les permitía elaborar sus prácticas.

- Intensificación de los mecanismos de evaluación continua (participación en clase y resolución de ejercicios) que ya se venían empleando.

- Adaptación de las herramientas de evaluación, tanto teóricas como prácticas, introduciendo cambios en el reparto de pesos con el objetivo de que la potencial ${ }^{2}$ no realización de un examen presencial no impidiese la superación de las asignaturas. En la Sección 4.5 se describen con mayor detalle estas modificaciones y, en particular, una de las actividades introducidas como herramienta de evaluación teórica.

- Incorporación de nuevas herramientas que permitiesen sustituir algunas de las actividades en clase, como la pizarra virtual compartida, gracias a la cual los estudiantes pudieron realizar ejercicios de manera colaborativa y síncrona.

Finalizado el curso, con un nivel de satisfacción que nos atreveríamos a calificar como bueno tanto por parte de los estudiantes como de los profesores teniendo en cuenta las dificultades afrontadas, nos planteamos aprovechar la experiencia adquirida para, de forma más pausada, continuar en la línea iniciada de adaptación de metodologías y herramientas. La meta es que el curso 2020/2021 pueda desarrollarse en completa normalidad indistintamente de que las sesiones conjuntas entre profesorado y estudiantes sean presenciales, en un aula, o bien a través de medios digitales.

En el resto de esta sección detallamos la combinación de metodologías de aprendizaje y herramientas en la que pretendemos apoyarnos durante el nuevo curso para conseguir la mencionada meta. Estas se han resumido esquemáticamente en la Figura 2 agrupándolas en cuatro bloques: estudio de materia teórica, realización de actividades prácticas, herramientas para facilitar la comunicación profesor-estudiante y entre estudiantes y sistemas de evaluación. En las siguientes subsecciones se detalla cada bloque.

\footnotetext{
${ }^{2}$ Por aquel entonces se pensaba que el confinamiento solo duraría unas pocas semanas, no hasta finalizar el curso.
} 


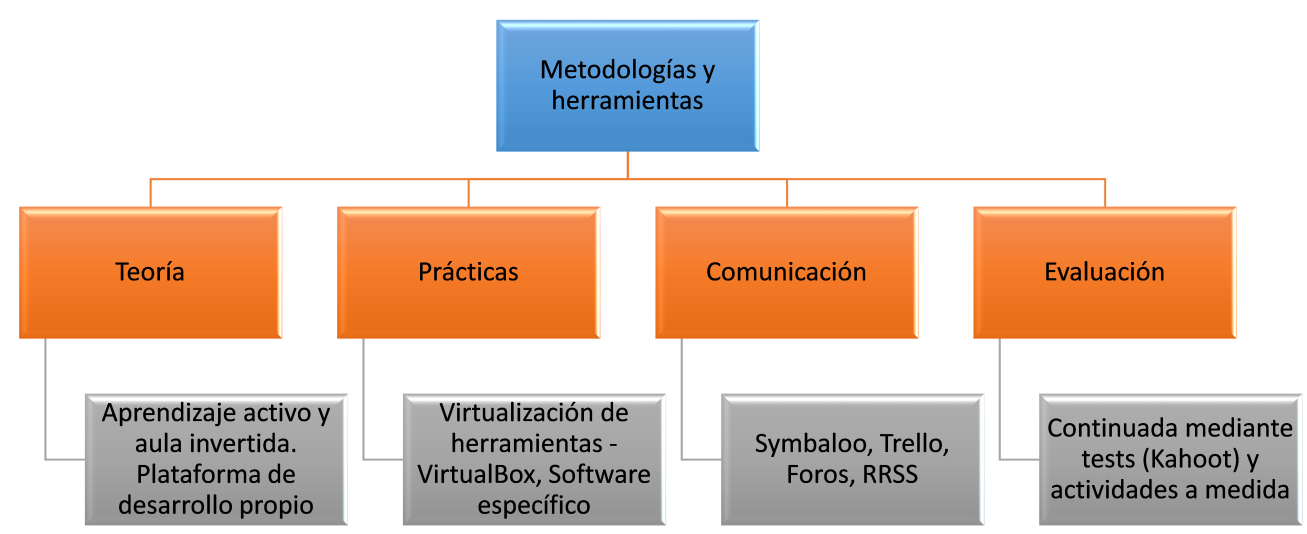

Figura 2. Resumen esquemático de las metodologías y herramientas

\subsection{Potenciación del aprendizaje activo durante el curso}

El aprendizaje activo consiste en ceder cierto nivel de control sobre el proceso al estudiante, incrementando su autonomía y favoreciendo la adquisición de conocimiento a través de la experiencia más que como un oyente pasivo, pasando así de espectador a convertirse en actor principal. A priori es una de las mejores maneras de aprovechar el tiempo de los estudiantes con el docente, que es el elemento esencial, usando la tecnología para potenciar el aprendizaje al permitir trasladar las tareas de bajo nivel cognitivo: estudio y repaso de temas mediante vídeos y otro tipo de documentos conjuntamente con la autoevaluación, al tiempo de trabajo autónomo.

El impacto que la introducción de técnicas de aprendizaje activo tiene en los resultados obtenidos por los estudiantes es un tema ampliamente estudiado, con cientos de publicaciones al respecto que informan tanto en sentido positivo como negativo. En [8] los autores llevan a cabo un meta-análisis de 225 de esos estudios centrándose en áreas STEM (Science, Technology, Engineering and Mathematics). De dichos estudios, la mayor parte indican una reducción en las tasas de suspensos tras la introducción de las mencionadas técnicas, así como una mejora en las calificaciones que se establece en un $6 \%$ de promedio. Al centrar los estudios seleccionados en el área de Ciencias de la Computación, que es la que nos interesa, los resultados positivos son prácticamente unánimes.

El sistema de créditos ECTS establece la cantidad de trabajo autónomo que los estudiantes han de desarrollar, siendo este entre 1.5 y 2 veces superior (15-20 horas por ECTS) al que se lleva a cabo de manera supervisada en clases de teoría y prácticas (10 horas por ECTS). Hasta ahora la mayoría de propuestas de aprendizaje activo tienen como contexto la clase o el laboratorio, siendo pocas las que ponen el foco en ese tiempo adicional en el que el estudiante ha de aprender por sí mismo. El mecanismo de aprendizaje autónomo más usual, tal y como se apunta en [17], es la relectura de diapositivas o apuntes, generalmente dedicando muchas horas a esta tarea en un intervalo de tiempo corto (el típico atracón antes de exámenes). Los autores de [17] 
realizan un extenso estudio del que se extraen fundamentalmente dos conclusiones: 1) el aprendizaje no mejora con la lectura repetitiva del material, pero sí lo hace mediante la recuperación de datos ya sabidos a través de tests y recursos similares, y 2) los conocimientos adquiridos se ven reforzados cuando hay un espaciamiento en el tiempo entre el estudio inicial y los posteriores, en contraposición al estudio en masa (muchas horas y mucho contenido) que es el más frecuente.

Los resultados de los dos estudios previos es lo que nos llevó a proponer mecanismos de aprendizaje activo dirigidos al estudio autónomo, pero guiados y coordinados con la materia impartida durante el periodo docente en lugar de para ser usados a la finalización del mismo. De entre las distintas herramientas existentes, las conocidas como flashcards [23] han demostrado ser especialmente eficientes. Estas se presentan al estudiante como un juego que estimula la recuperación de los conocimientos que ya ha obtenido, factor que los refuerza según se indica en [17]. La planificación y seguimiento automáticos, a través de una plataforma adecuada, ofrecerá estas flashcards en el momento y con la frecuencia adecuados.

El objetivo principal del proyecto de innovación docente que teníamos en marcha cuando afrontamos esta situación de cambio a docencia remota era evaluar empíricamente si era posible mejorar el proceso de aprendizaje, y los resultados finales obtenidos por los estudiantes, mediante la introducción de técnicas de aprendizaje activo. Este objetivo se compondría de los siguientes hitos:

- Diseño y desarrollo de una plataforma web a través de la cual se pondrían a disposición de los estudiantes distintos recursos específicos que demanden una actitud activa: flashcards, vídeos con cuestionarios, etc. La plataforma realizará un seguimiento de la interactuación de los estudiantes con la misma: recursos que emplean, tiempo dedicado y resultados obtenidos.

- Preparación de los materiales didácticos a incluir en la plataforma por parte de los profesores implicados.

- Puesta en marcha experimental de la plataforma a lo largo de un curso académico, durante el cual una parte de los estudiantes de una asignatura tendrían acceso a la misma. El resto del alumnado servirían como grupo de control o referencia. Esta es la fase en que nos encontrábamos en marzo de 2020.

- Realización de uno o más test no evaluables al conjunto total de estudiantes que, conjuntamente con los resultados de las pruebas evaluables habituales, servirán como índices a analizar.

- Al finalizar el curso académico, análisis de los índices previos y de los datos registrados en la plataforma a fin de determinar si existen diferencias significativas entre los resultados obtenidos por los estudiantes dependiendo de si han participado o no en la experiencia, así como según el uso que han hecho de los recursos existentes en la plataforma.

Incluir un enfoque lúdico en el proceso de aprendizaje (gamificación [24,25]) habitualmente sirve como estímulo para el estudiante, pudiéndose llegar al nivel de competición entre ellos. El uso de las flashcards [23] a modo de juego, con una puntuación en función de la velocidad y el acierto en las respuestas a los test de autoevaluación, nos pareció una alternativa interesante. 


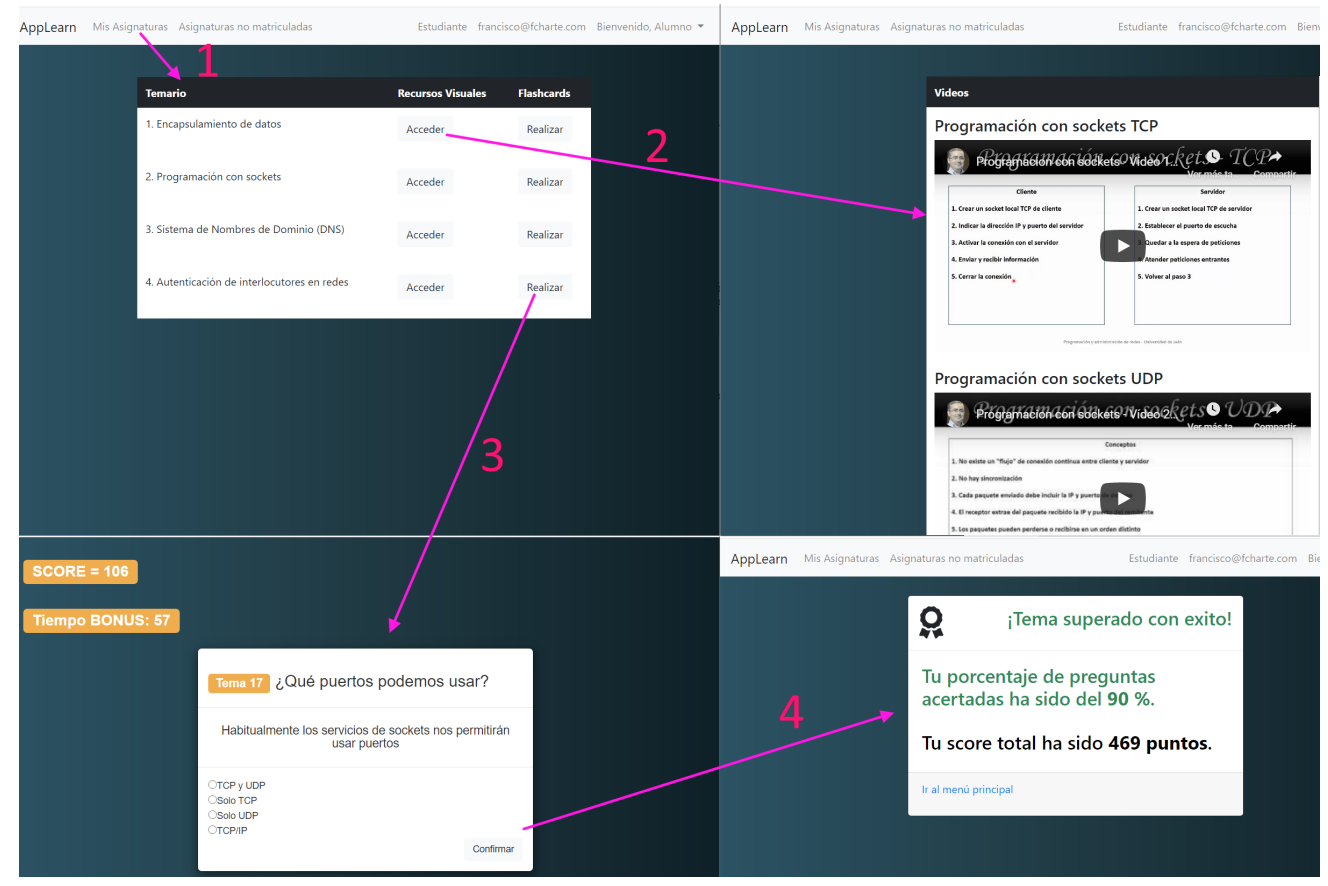

Figura 3. Vistas de la aplicación de aprendizaje activo para el estudiante

Asociado a este proyecto se desarrolló una plataforma específica accesible públicamente (https://activelearn.ml) con un doble objetivo: facilitar a los estudiantes los recursos de aprendizaje y herramientas de autoevaluación, por una parte, y permitir a los docentes recolectar toda la información relacionada con el uso de la plataforma, tanto individual como agregada, a fin de poder evaluar su impacto en la evolución de los estudiantes. En la Figura 3 se muestran las vistas principales disponibles para el estudiante, con la secuencia de uso habitual compuesta de los siguientes pasos:

1. Acceso a la lista de asignaturas y selección de aquella con la que quiere trabajar, obteniendo la lista de temas disponibles.

2. Acceso a los recursos disponibles para un tema, principalmente vídeos en los que el profesorado de la asignatura expone conceptos de la materia.

3. Acceso a la herramienta de autoevaluación mediante flashcards. La aplicación cambia a un modo de pantalla completa en la que el estudiante solo ve su puntuación, el bonus que tiene por tiempo, las preguntas y respuestas y la confirmación de si ha fallado o acertado a medida que avanza.

4. Rúbrica con los resultados de la autoevaluación indicando el porcentaje de aciertos y también la puntuación o score final que permitiría al estudiante comparar sus resultados con los obtenidos por sus compañeros.

Para los docentes implicados, la puesta en marcha de este proyecto ha supuesto las siguientes actividades adicionales: 


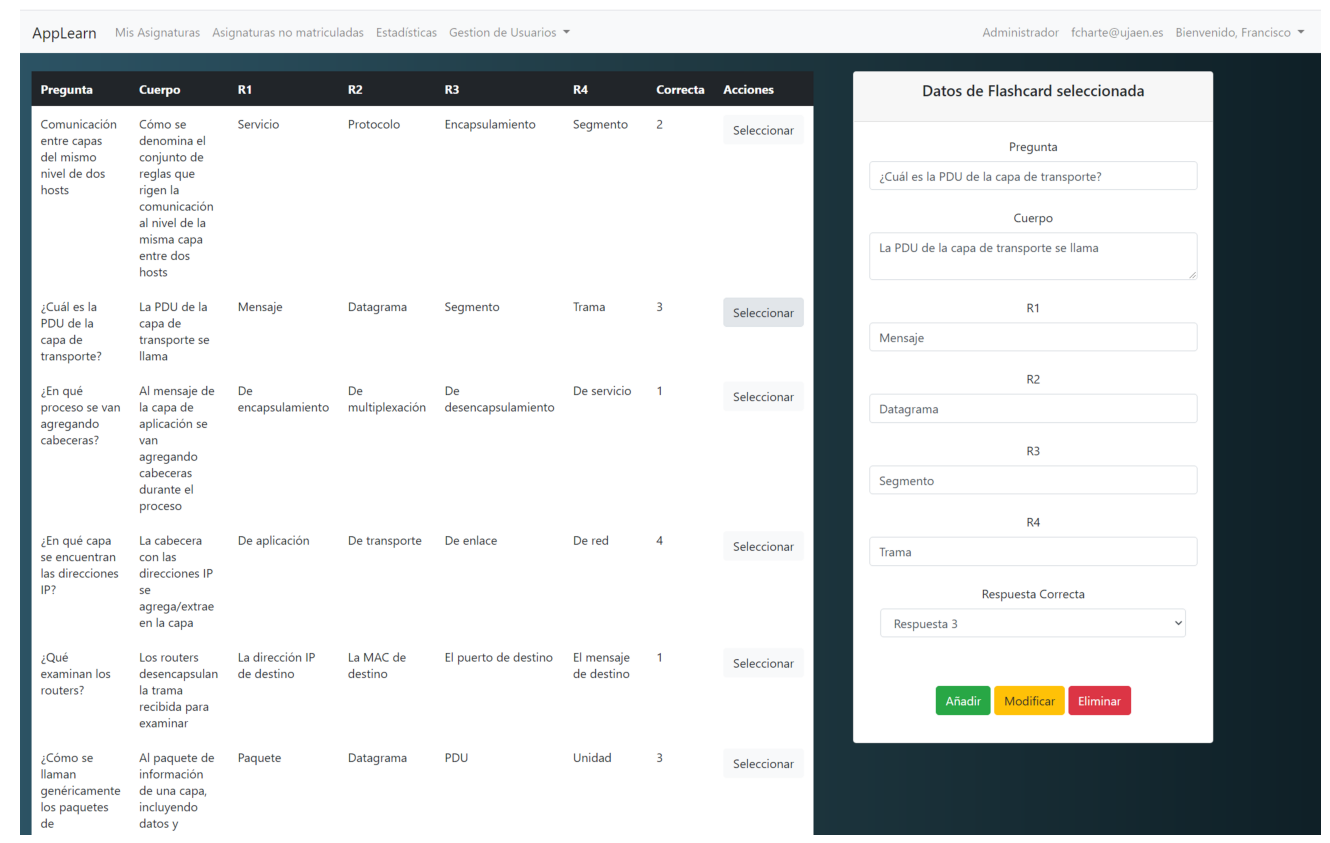

Figura 4. Panel para la composición de las flashcards por parte del profesor

- Elaboración de material audiovisual sobre las materias más importantes de la asignatura, con una estructura similar a la de la clase magistral pero en un soporte al que el estudiante puede acceder siempre que le interese.

- Diseño del conjunto de flashcards asociadas a cada tema, empleando para ello la herramienta mostrada en la Figura 4 que forma parte de la plataforma web antes mencionada.

- Análisis estadístico de los datos recopilados por la plataforma (véanse las Figuras 5 y 6), entre los que se incluyen la frecuencia de uso de los recursos por parte de cada estudiante, las ratios de acierto tanto por estudiante como por flashcard, etc.

Desde la perspectiva del profesor, la información que le aporta esta plataforma ampliaría las posibilidades de personalización del aprendizaje en función de las capacidades y resultados de cada estudiante, algo que es posible cuando se trabaja con grupos reducidos. Para grupos grandes, la clave posiblemente esté en que el profesor, a través de las flashcards, plantee las preguntas apropiadas para lograr que el estudiante interactúe con el contenido, no limitándose a ver el vídeo sin más, sino estudiándolo para encontrar las respuestas a esas preguntas. En este sentido, esta herramienta también puede representar un estímulo a la colaboración entre los estudiantes con el objetivo de elaborar lo aprendido a través de los recursos facilitados, poniendo en común conceptos e ideas.

Un hecho importante es que aunque los profesores pueden ir añadiendo recursos y sus correspondientes flashcards a lo largo del curso, el estudiante tiene la opción de 


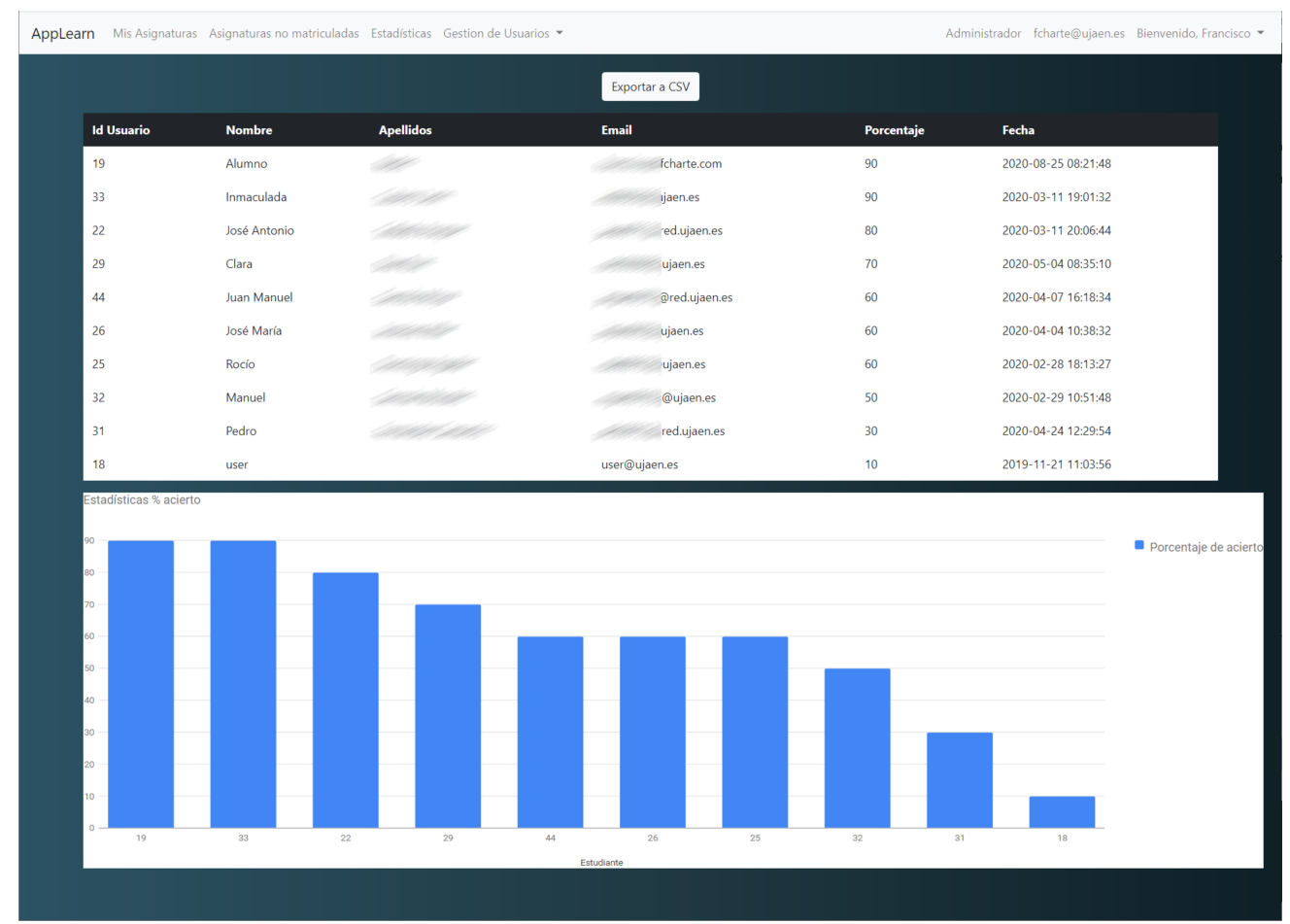

Figura 5. Algunos de los resúmenes estadísticos facilitados por la plataforma ActiveLearn (I)

acceder a los ya disponibles tantas veces como lo precise y sin limitaciones de fechas $\mathrm{u}$ horarios. Aunque es algo que queda en gran medida fuera del control del docente, hay que tener en cuenta el bienestar personal del estudiante ya que, por regla general, el estrés es un obstáculo para la concentración. Una forma de reducir ese estrés es permitirle saber en todo momento dónde se encuentra en el proceso de aprendizaje de una materia, una necesidad que la plataforma descrita es capaz de satisfacer.

\subsection{El aula invertida como complemento de la clase magistral}

Las metodologías relacionadas con el aula invertida [14] son planteadas en muchos casos como una sustitución de la habitual clase magistral. En nuestra opinión dicha metodología, junto con otras estrechamente relacionadas como las ya mencionadas del aprendizaje basado en proyectos, pueden actuar también como un complemento.

En nuestro caso el objetivo que nos planteamos es emplear la plataforma de aprendizaje activo descrita en la sección previa como vía para que los estudiantes adquieran las nociones y conceptos generales sobre la materia, aquellos que no precisan de un análisis demasiado profundo y que esencialmente son objeto de los métodos de estudio clásico, aunque ahora a través de medios digitales. Esto permitirá aliviar la necesidad de clases magistrales durante las horas compartidas entre docentes y estudiantes, si bien estas 


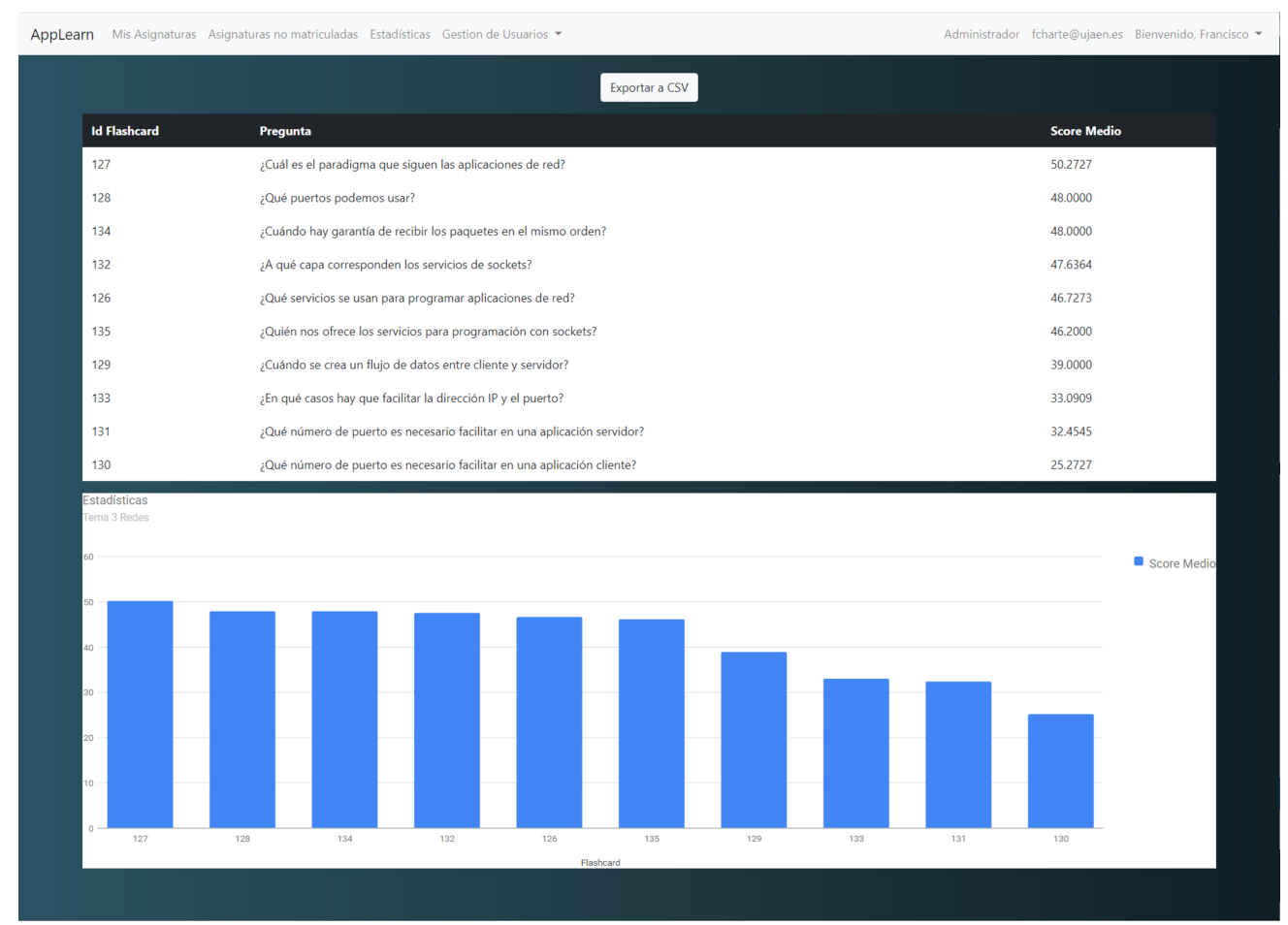

Figura 6. Algunos de los resúmenes estadísticos facilitados por la plataforma ActiveLearn (II)

seguirían siendo imprescindibles para cubrir aspectos concretos más especializados o con un mayor nivel de complejidad. El resto del tiempo se dedicará a discusiones de temas, que los estudiantes habrán preparado a través de la plataforma de aprendizaje activo y otros materiales complementarios que pudieran facilitarse a través de la misma o un LMS tradicional, así como a la resolución de problemas y ejercicios.

Los medios para facilitar esa aula invertida no difieren en exceso dependiendo de la situación que sea preciso afrontar a lo largo del curso. En modalidad presencial, en el aula universitaria, la comunicación resulta más natural e inmediata y la tradicional pizarra sigue siendo probablemente la mejor herramienta de apoyo. En modalidad remota la clase síncrona se desarrollará mediante vídeoconferencia, mientras que la pizarra física es sustituida por una virtual compartida entre profesor y estudiantes, de forma que es posible la resolución individual o colaborativa de las actividades planteadas.

\subsection{Virtualización del material de laboratorio}

La adaptación a un sistema de enseñanza remoto resulta relativamente sencillo en cuanto a las herramientas que se precisan para cubrir los aspectos teóricos, muchas de la cuales ya se han citado: sala de videoconferencia, pizarra virtual compartida, etc., y otras que se mencionarán en la siguiente sección. Además, son aplicaciones en gran medida útiles indistintamente de cuál sea la materia concreta de estudio. 
En contraposición, la adecuación de las prácticas ha supuesto en general un esfuerzo mayor. En nuestro caso, centrándonos en dos asignaturas concretas, la solución ha venido a través de la virtualización y simulación/emulación de los elementos empleados habitualmente en el laboratorio. En titulaciones de grado y máster relacionadas con la informática esta es una alternativa que puede resultar útil en la mayoría de los casos.

Nuestra asignatura Programación y administración de redes cuenta con actividades prácticas en las que los estudiantes conectan físicamente varios ordenadores en redes locales, empleando el cableado y hardware habitual, y llevan a cabo distintas configuraciones y pruebas. El traslado de este tipo de actividades al equipo del estudiante, para que pueda completar sus prácticas desde casa, ha conllevado fundamentalmente una dificultad: ajustar las herramientas a los recursos hardware limitados de dichos equipos que, en ocasiones, eran muy serías en cuanto a memoria disponible y potencia de procesamiento.

La solución fue crear una imagen de máquina virtual con los elementos necesarios para completar las prácticas pero eliminando todos los aspectos innecesarios: interfaz gráfica de usuario, aplicaciones de productividad, etc., consiguiendo así su funcionamiento con solo $256 \mathrm{MB}$ de memoria RAM y un bajo consumo de uso de procesador. Esto permite a los estudiantes iniciar hasta cuatro instancias de la máquina virtual, simulando el uso de otros tantos ordenadores en el laboratorio, procediendo a su configuración con la única diferencia de que no se emplea hardware físico sino virtual (adaptadores de red virtuales). Además del software en sí, también fue preciso facilitar a los estudiantes las instrucciones necesarias para llevar a cabo la configuración de virtualización de forma que pudieran consultarlo siempre que lo necesitasen (https : //www . youtube.com/watch?v=jNUQsmXyj2Q).

Otra de las asignaturas que teníamos en curso cuando se produjo el confinamiento era Sistemas empotrados y ubicuos, cuyas prácticas se apoyan en parte en el uso de unas placas de entrenamiento para desarrollo de soluciones basadas en FPGA [26], empleando para ello el software facilitado por el fabricante, en particular Altera Quartus y Lattice IceCube. Este tiene la particularidad de ser bastante pesado, por una parte, y de requerir configuración de licencias, por otro, aspectos ambos resueltos en nuestros laboratorios.

En este caso la solución fue recurrir a herramientas que permiten trabajar con lenguajes de descripción de hardware, como VHDL y Verilog, sin necesidad de herramientas específicas de un fabricante, como es el caso de EDA Playground (www. edaplayground.com) o la combinación de aplicaciones Yosys (www.clifford.at/yosys), Apio (github.com/FPGAwars/apio) y Icestudio (icestudio.io). La mayor limitación de todas ellas es que, en general, no es posible probar las soluciones desarrolladas si no se lleva a cabo un despliegue en una placa FPGA. Para suplir esta carencia se recurrió al emulador Deeds (www.digitalelectronicsdeeds.com) que, a través de su interfaz gráfica, permite interactuar con las entradas y salidas del circuito diseñado y comprobar su funcionamiento.

Para el curso 2020/2021 los mecanismos de virtualización, incluso si la realización de las prácticas de forma presencial fuese posible, seguirán empleándose a fin de que los estudiantes puedan planificar e incluso llegar a desarrollar sus actividades prácticas empleando una configuración idéntica o muy cercana a la que tendrá luego en el laboratorio. 


\subsection{Comunicación durante y fuera de las clases}

Un aspecto que en ocasiones se minusvalora durante la actividad presencial en el aula, probablemente porque siempre se ha dado por hecha, es la comunicación tanto durante el desarrollo de las clases como fuera de estas. Por una parte es importante la comunicación profesor-estudiante, pero sobre todo, como se apuntaba anteriormente, entre los propios estudiantes.

Los mecanismos usados tradicional para hacer posible esa comunicación, especialmente en el caso estudiante-profesor, son el correo electrónico y las tutorías. Los foros en el LMS son también una vía de contacto, tanto con el profesor como entre los propios estudiantes. No obstante, no hemos de ignorar que los alumnos están habituados a emplear sobre todos redes sociales [27] para comunicarse entre ellos, medio que les resulta más natural.

Nuestra experiencia en este área, concretamente con el uso de Twitter (https: //twitter.com/EPSJ_PAR) a través de una cuenta específica para la asignatura como se aprecia en la Figura 7, ha sido positiva encontrando una buena acogida por parte de los estudiantes. Habitualmente empleamos este canal para hacer llegar avisos, enlaces a herramientas y noticias relacionadas con la asignatura, que los estudiantes difunden a través de sus propias cuentas. En menor medida, este medio también es empleado por ellos para plantear cuestiones que son respondidas tanto por sus compañeros como por el profesorado.

La comunicación entre los estudiantes en situación presencial es un punto de apoyo que pierden en gran medida en caso de que la situación impida la asistencia al aula. Con el objeto de facilitar tanto la compartición de recursos entre ellos como la coordinación, por ejemplo a la hora de llevar a cabo actividades colaborativas, es fundamental contar con las herramientas adecuadas. En nuestro caso nos decantamos fundamentalmente por dos opciones concretas, ambas aplicaciones web accesibles desde cualquier dispositivo con conexión a Internet:

- Symbaloo - www.symbaloo.com: a través de sus WebMix, esta aplicación permite a los estudiantes recopilar enlaces a recursos de todo tipo alojados en la web u otro tipo de fuentes, como RSS, podcast, etc. Cada WebMix puede ser privado o público y su configuración es muy flexible, de forma que es posible agrupar las losetas que representan los recursos según las necesidades, diferenciar grupos por colores, establecer losetas que dan paso a grupos de losetas, etc. En la Figura 8 se muestra el WebMix inicial que se facilitaría a los estudiantes de la asignatura Programación y administración de redes, conteniendo enlaces de referencia como IEEE, IETF y W3C. Sobre ella, los propios estudiantes pueden ir agregando otros recursos de manera colaborativa. Esto les permite contar con un conjunto de materiales de estudio que en gran parte ellos mismos han seleccionado por su utilidad para el aprendizaje de la materia o la resolución de problemas asociados a la misma.

- Trello - trello.com: esta herramienta lleva siendo usada desde hace años por algunos de los autores para la coordinación de trabajos propios, pero resulta también adecuada para la coordinación de tareas en las que trabajarán los estudiantes por grupos. Como se aprecia en la Figura 9, el profesor puede crear en la pizarra del 


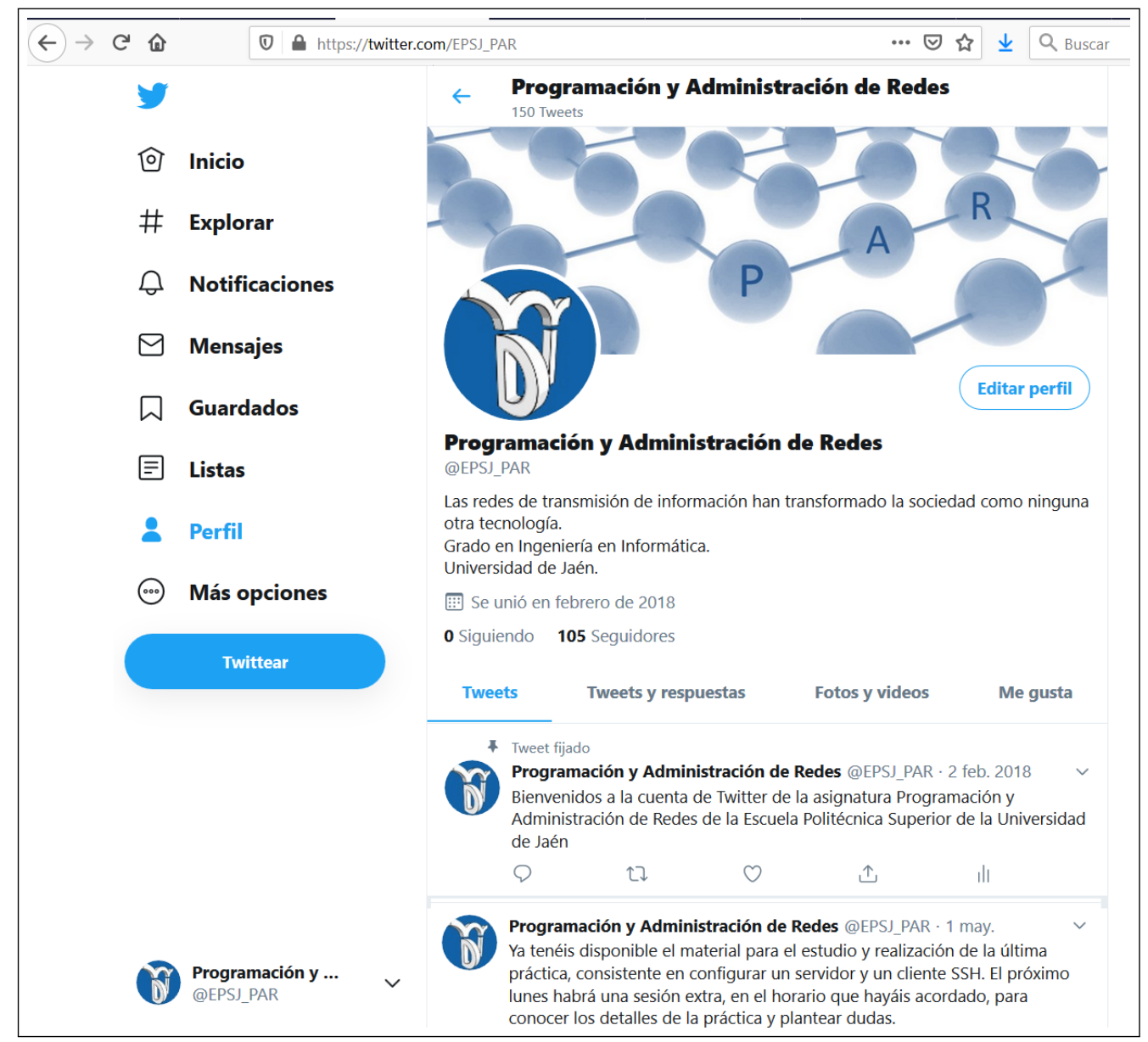

Figura 7. Cuenta de Twitter para una de las asignaturas impartidas por los autores

curso actual diferentes listas de tareas, cada una de ellas compuesta de un conjunto de parámetros: recursos asociados a la tarea, miembros de participarán en la misma, hitos a ir superando a lo largo del tiempo, vencimiento de la tarea, etc. Las opciones de Trello permiten, no solo que los estudiantes se coordinen entre sí, sino que también da oportunidad al docente de supervisar el trabajo desarrollado por cada uno de ellos.

La selección de utilidades generales para la compartición y coordinación, como las ya citadas, no es óbice para que durante el desarrollo de tareas concretas se recurra a otras herramientas más específicas, como son Github/Gitlab (github.com/gitlab. ujaen.es) para el desarrollo colaborativo de proyectos, Overleaf (www.overleaf .com) o Google Docs (docs.google.com/) para la composición de documentos por grupos, etc. 


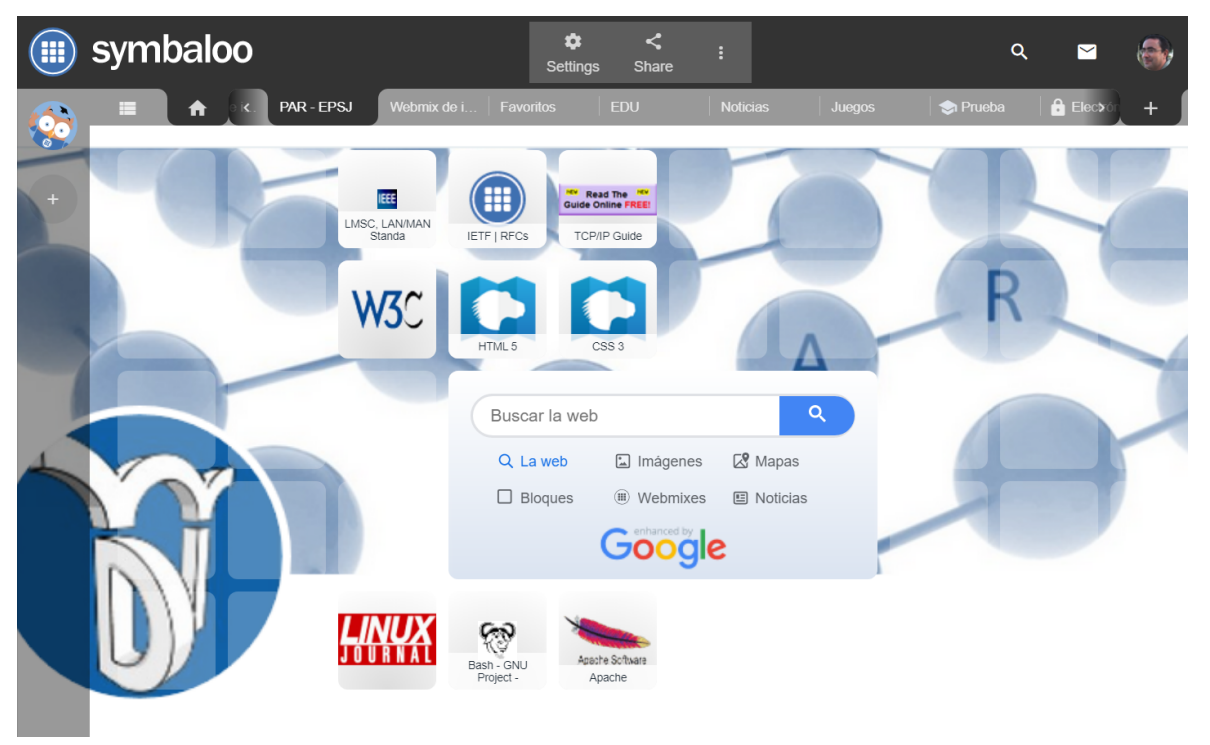

Figura 8. Webmix de inicio para la asignatura Programación y administración de redes

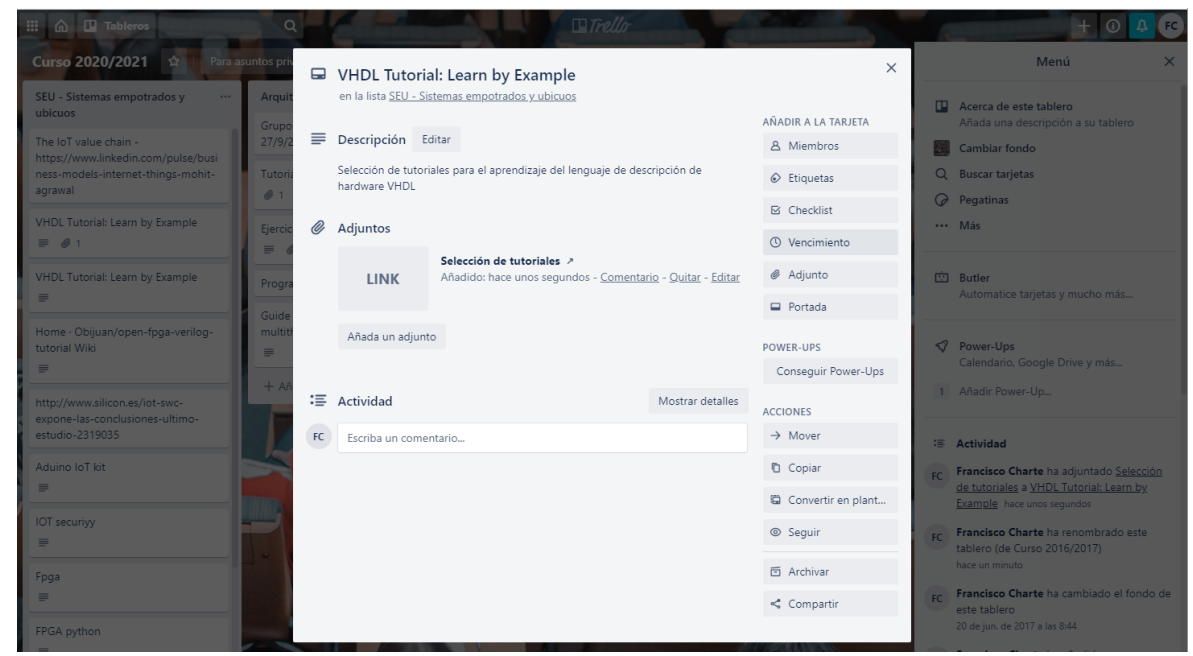

Figura 9. Configuración de una tarea en Trello, estableciendo miembros que participarán y fecha de vencimiento

\subsection{Técnicas de evaluación alternativas}

La adaptación de los sistemas de evaluación a una modalidad no presencial ha sido probablemente el desafío que más discusiones y diferencias ha generado entre todas las partes, especialmente entre los estudiantes y los docentes cuando estos últimos han 
optado por recurrir a un sistema de proctoring [28] que, en general, no es bienvenido por los primeros.

Partimos de una situación general de desconfianza entre las partes, en la que el profesor actúa a veces más como vigilante que como docente, en un sistema rígido que da pocas oportunidades a la innovación en este sentido. Se prima mucho más la evaluación individual basada en memorizar conocimientos que otros aspectos, tanto o más importantes, como es la capacidad del trabajo en grupo y para la resolución de problemas.

Tenemos herramientas para cambiar esto, como puede ser la evaluación mediante aprendizaje basado en proyectos $[29,30]$. Aunque muchos asocien esta metodología con otras más recientes, como el aula invertida, y tengan la noción de algo novedoso y no probado, el aprendizaje basado en proyectos viene utilizándose desde hace casi cien años.

Nuestra propuesta es el uso combinado de múltiples vías de evaluación con un reparto de pesos adecuado. Concretamente recurriremos a las siguientes herramientas:

- Evaluación del aprendizaje activo y continuado mediante la realización de tests frecuentes en clase usando la herramienta Kahoot! (kahoot.com).

- Evaluación basada en proyectos específicos que potencien las habilidades de recopilación, curación y elaboración de contenidos relacionados con la materia de estudio (descrito en detalle después).

- Evaluación de habilidades prácticas mediante un sistema dual que, por una parte, incluye la realización síncrona de tareas bajo las indicaciones del profesor, ya sea en el aula o mediante la compartición del escritorio del estudiante, y por otra la realización de test sobre conceptos prácticos.

- Evaluación de la capacidad de resolución de problemas teórico/prácticos, ya sea mediante un examen tradicional realizado de forma síncrona o con metodologías basadas en proyectos [31].

A modo de ejemplo de actividad de evaluación basada en proyectos, presentamos a continuación la propuesta Vídeos 1 tema en 1 minuto. Se trata de una tarea creativa, de alto nivel cognitivo, que requiere de guía y supervisión por parte del profesor, aparte de representar una técnica de evaluación alternativa al tradicional examen escrito. La infografía de presentación es la mostrada en la Figura 10 y a continuación se detalla la información que los estudiantes obtienen al interactuar con ella Genial.ly ${ }^{3}$.

\section{Visión general de la actividad ofrecida a los estudiantes}

- Objetivos: esta actividad forma parte de la evaluación de contenidos teóricos de la asignatura, siendo su principal objetivo que el/la estudiante refuerce su conocimiento sobre un tema específico, mejore sus capacidades de comunicación y favorezca el trabajo en grupo entre estudiantes. Una vez asignado un tema, cada estudiante recopilará información sobre él, curará el contenido con ayuda de otros estudiantes y elaborará un vídeo explicando lo esencial sobre dicho tema.

\footnotetext{
${ }^{3}$ https://view.genial.ly/5eec64a9b4bde70d10f15ad1/horizontal-infographic-review-curacionde-contenidos-en-el-aula-programacion-y-administracion-de-redes
} 


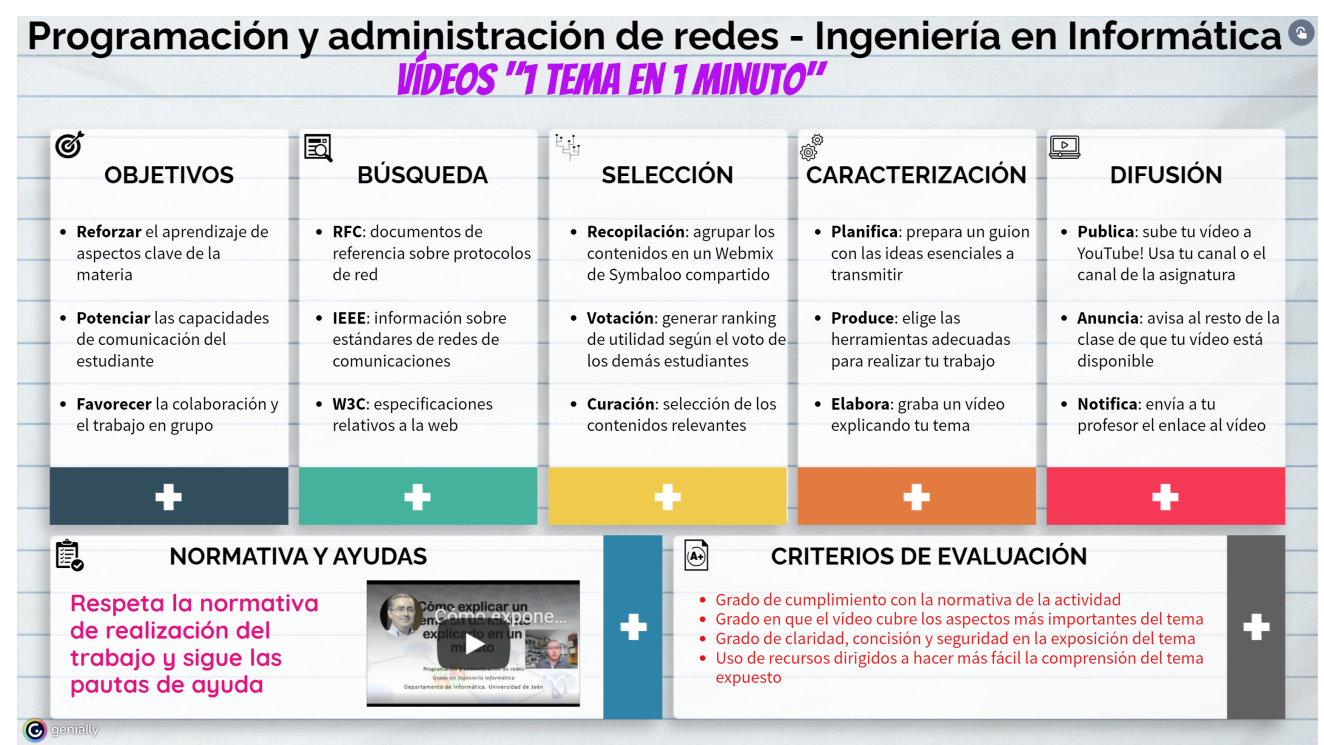

Figura 10. Presentación dinámica de la actividad Vídeos 1 tema en 1 minuto

- Búsqueda: usa fuentes de información fiables, como son los RFC (Request For Comments) oficiales y las especificaciones publicadas por IEEE y W3C. También puedes recurrir a libros y artículos científicos.

- Selección: comparte tus contenidos con el resto de estudiantes en el Webmix compartido de la asignatura. Colabora en la curación de los contenidos aportados por los demás estudiantes. Selecciona de tus contenidos los mejor valorados por tus compañeros/as.

- Caracterización: usando los contenidos ya curados de la fase anterior acerca del tema de interés, planifica un guion que te permita explicar lo esencial de una forma comprensible. Usa las herramientas que te resulten más cómodas para generar un vídeo con tu explicación.

- Difusión: una vez creado el vídeo, súbelo a tu canal de YouTube u otro servicio de publicación de vídeos como vídeo privado y facilita el enlace a los profesores. También puedes usar el canal en YouTube de la asignatura si prefieres hacerlo público, de esta forma a continuación podrás promocionar tu trabajo anunciándolo a los demás estudiantes. No olvides notificar a tu profesor la disponibilidad del vídeo para que pueda evaluarlo.

\section{Normativa y consejos mostrados a los estudiantes}

Los trabajos realizados en esta actividad deben de cumplir la siguiente normativa:

- Cada estudiante realizará un vídeo sobre el tema asignado.

- En el vídeo debe aparecer el estudiante durante la mayor parte del tiempo. 
- El vídeo tendrá una duración de entre 1:00 y 1:30 minutos.

- El vídeo comenzará con una portada de unos segundos indicando su título o tema.

- El vídeo deberá generarse en formato MP4.

- El vídeo se entregará mediante la plataforma ILIAS ${ }^{4}$.

Condensar un cierto concepto o tema en un minuto nos forzará a extraer lo esencial, lo importante, para lo cual es indispensable conocer muy a fondo dicho tema. Se os recomienda lo siguiente:

1. Comienza recopilando toda la información posible sobre el tema en cuestión, asegurándote de que usas fuentes fidedignas como son libros, especificaciones o artículos científicos.

2. Estudia a fondo el material que hayas obtenido, crea esquemas que resuman las ideas y extrae de ellas los aspectos más vitales para comprender el tema a exponer. Si lo haces sobre papel te resultará más fácil.

3. Prepara un guion con entre 4 y 6 puntos, centrando cada uno de ellos en un aspecto de los identificados en el paso previo. Ten en cuenta que tendrás entre 10 y 15 segundos para explicar cada punto.

4. Prepara las herramientas que vas a usar para la grabación, puedes usar tu móvil o una webcam, sin olvidar tu guion y un cronómetro que puedas estar viendo en todo momento.

No es imprescindible grabar el vídeo en una sola toma. Puedes grabar distintas partes, incluso usando diferentes dispositivos, por ejemplo, para recurrir a recursos que hagan más fácil la comprensión del tema grabándolos con el móvil. También puedes usar diagramas o cualquier material que hayas creado, en papel o en el ordenador.

\section{Criterios de evaluación}

Los profesores calificarán los trabajos entregados por los estudiantes atendiendo a los siguientes criterios:

- Grado de cumplimiento con la normativa de la actividad antes expuesta

- Grado en que el vídeo cubre los aspectos más importantes del tema correspondiente

- Grado de claridad, concisión y seguridad en la exposición del tema

- Uso de recursos dirigidos a hacer más fácil la comprensión del tema expuesto

\section{Resultados de la experiencia}

En el curso 2019/2020 ya finalizado, durante la fase de confinamiento, realizamos esta propuesta ofreciendo 100 temas diferentes relacionados con la asignatura Programación y administración de redes, tantos como estudiantes matriculados en la misma. De ellos se asignaron 92 a estudiantes que lo solicitaron, de los cuales finalmente se entregaron y evaluaron 90. La experiencia fue gratificante para una buena parte de los estudiantes, algo que quedó plasmado en la creatividad que desplegaron en la realización de sus vídeos. Es una vía de evaluación que pretendemos seguir empleando en el próximo curso, conjuntamente con las otras ya mencionadas, con independencia de la situación y nivel de presencialidad.

${ }^{4}$ ILIAS es el LMS usado en la Universidad de Jaén 


\section{Conclusiones}

El sistema educativo tiene una gran inercia que viene propiciada de su propia tradición como componente socicultural y no es posible cambiarlo de un día para otro, sería como intentar girar bruscamente la dirección de un camión a toda velocidad. Por ello es preciso ir iniciando ese giro cuanto antes, suave pero constantemente, favoreciendo una adaptación que se antoja será imprescindible en el futuro más inmediato.

Hace 20 años los estudiantes usaban la biblioteca para consultar libros, mientras que en la actualidad recurren mucho más a Google, YouTube y herramientas digitales. Por ello localizar los recursos de aprendizaje ya no es tan importante como antes, en la actualidad el acceso a recursos digitales es mucho más sencillo, debiéndose poner el énfasis en la construcción de conocimiento a partir de esas fuentes de datos.

Desde nuestra perspectiva el uso de nuevas metodologías y herramientas, sobre todo tecnológicas, no va en contra de la presencialidad, la cual es fundamental para aspectos como la comunicación, o la tradicional clase magistral. Lo ideal es tomar lo mejor de los dos mundos, el digital y el presencial, para dar lugar a un contexto de aprendizaje mixto, un ecosistema que permita al estudiante continuar con independencia de cuál sea la situación concreta en cada momento y los cambios que puedan producirse a lo largo del tiempo. Es aquí donde elementos como la pizarra digital compartida, la virtualización de medios de laboratorio, el aprendizaje activo y basado en proyectos o la innovación en los modelos de evaluación, entre otros aspectos descritos en este artículo, jugarán un papel fundamental de cara al éxito en el proceso de enseñanza/aprendizaje.

\section{Referencias}

1. M. de Educación y Ciencia secretaría de Estado de Universidades e Investigación, Propuestas para la renovación de las metodologías educativas en la Universidad, Tech. rep. (2006).

2. L. J. C. Rodríguez, Efectos del coronavirus en el sistema de enseñanza: aumenta la desigualdad de oportunidades educativas en españa., Revista de Sociología de la EducaciónRASE 13 (2) (2020) 114-139.

3. N. G. Fernández, M. L. R. Moreno, J. R. Guerra, Brecha digital en tiempo del covid-19, Revista Educativa HEKADEMOS (28) (2020) 76-85.

4. J. Silva, Un modelo pedagógico virtual centrado en las e-actividades, Revista de Educación a Distancia (RED) (53).

5. Y. d. V. Ramírez León, et al., Adaptación del diseño de unidades didácticas a estilos de aprendizaje en entornos virtuales de enseñanza-aprendizaje.

6. W. R. Zambrano, V. H. M. García, A. V. M. García, Nuevo rol del profesor y del estudiante en la educación virtual, Dialéctica: Revista de investigación (26) (2010) 51-62.

7. L. W. Anderson, L. A. Sosniak, Bloom's taxonomy, Univ. Chicago Press Chicago, IL, 1994.

8. S. Freeman, S. L. Eddy, M. McDonough, M. K. Smith, N. Okoroafor, H. Jordt, M. P. Wenderoth, Active learning increases student performance in science, engineering, and mathematics, Proceedings of the National Academy of Sciences 111 (23) (2014) 84108415.

9. P. C. Blumenfeld, E. Soloway, R. W. Marx, J. S. Krajcik, M. Guzdial, A. Palincsar, Motivating project-based learning: Sustaining the doing, supporting the learning, Educational psychologist 26 (3-4) (1991) 369-398. 
10. M. A. Albanese, S. Mitchell, et al., Problem-based learning: A review of literature on its outcomes and implementation issues, ACADEMIC MEDICINE-PHILADELPHIA- 68 (1993) 52-52.

11. J. Hattie, Visible learning for teachers: Maximizing impact on learning, Routledge, 2012.

12. J. Hattie, The applicability of visible learning to higher education., Scholarship of teaching and Learning in Psychology 1 (1) (2015) 79.

13. D. M. Fetterman, S. J. Kaftarin, S. J. Kaftarian, A. Wandersman, Empowerment evaluation: Knowledge and tools for self-assessment and accountability, Sage, 1996.

14. P. M. DeWitt, Flipping leadership doesn't mean reinventing the wheel, Corwin Press, 2014.

15. J. Biggs, Enhancing teaching through constructive alignment, Higher education 32 (3) (1996) 347-364.

16. G. T. Brown, G. H. Hirschfeld, Students' conceptions of assessment: Links to outcomes, Assessment in Education: Principles, Policy \& Practice 15 (1) (2008) 3-17.

17. R. A. Bjork, J. Dunlosky, N. Kornell, Self-regulated learning: Beliefs, techniques, and illusions, Annual review of psychology 64 (2013) 417-444.

18. H. Frick, J. Birt, J. Waters, Enhancing student engagement in large management accounting lectures, Accounting \& Finance 60 (1) (2020) 271-298.

19. I. Palomares, M. Espinilla, L. Martínez, Implantation and evaluation of ICT resources oriented to selflearning and self-assessment, in: International Conference Interactive Computer Aided Learning, IEEE, 2012, pp. 1146-1149.

20. M. S. Ibarra Sáiz, G. Rodríguez Gómez, Aprendizaje autónomo y trabajo en equipo: reflexiones desde la competencia percibida por los estudiantes universitarios., Revista electrónica interuniversitaria de Formación del profesorado 14 (4).

21. G. L. Placencia, Los vídeo-tutoriales en la educación universitaria del siglo xxi, Revista Iberoamericana de Producción Académica y Gestión Educativa 2 (3).

22. S. Waite, B. Davis, Developing undergraduate research skills in a faculty of education: Motivation through collaboration, Higher Education Research \& Development 25 (4) (2006) 403-419.

23. R. Schmidmaier, R. Ebersbach, M. Schiller, I. Hege, M. Holzer, M. R. Fischer, Using electronic flashcards to promote learning in medical students: retesting versus restudying, Medical education 45 (11) (2011) 1101-1110.

24. M. Espinilla, A. Fernandez, J. Santamaríaa, A. J. Rivera, Gamificación en procesos de auto-entrenamiento y auto-evaluación, Enseñanza y aprendizaje de ingeniería de computadores: Revista de Experiencias Docentes en Ingeniería de Computadores 6.

25. D. Dicheva, C. Dichev, G. Agre, G. Angelova, Gamification in education: A systematic mapping study., Journal of Educational Technology \& Society 18 (3).

26. F. Charte Ojeda, M. Espinilla Estévez, A. J. Rivera Rivas, F. J. Pulgar Rubio, Uso de dispositivos FPGA como apoyo a la enseñanza de asignaturas de arquitectura de computadores, Enseñanza y aprendizaje de ingeniería de computadores: Revista de Experiencias Docentes en Ingeniería de Computadores (7) (2017) 37-52.

27. B. C. Judd, C. A. Graves, Cellular stem: Promoting interest in science, technology, engineering, and math education using cellular messaging, cloud computing, and web-based social networks, in: 2012 12th IEEE/ACM International Symposium on Cluster, Cloud and Grid Computing (ccgrid 2012), IEEE, 2012, pp. 799-804.

28. K. Hylton, Y. Levy, L. P. Dringus, Utilizing webcam-based proctoring to deter misconduct in online exams, Computers \& Education 92 (2016) 53-63.

29. Y. Doppelt, Implementation and assessment of project-based learning in a flexible environment, International journal of technology and design education 13 (3) (2003) 255-272. 
30. Y. Gülbahar, H. Tinmaz, Implementing project-based learning and e-portfolio assessment in an undergraduate course, Journal of Research on Technology in Education 38 (3) (2006) 309-327.

31. A. Badia, C. García, Incorporación de las TIC en la enseñanza y el aprendizaje basados en la elaboración colaborativa de proyectos, International Journal of Educational Technology in Higher Education 12 (3) (2006) 42-53. 